\title{
Discussion des Communications XV-XVII
}

M. Mahaim: Je remercie très vivement les quatre orateurs qui viennent de nous documenter si brillamment sur Les Méthodes d'Examen des Cardiopathíes Congénitales. J'adresse un Temerciement special à nos hôtes étrangers, MM. McMichael et Mannheimer, qui se sont ingéniés à faire tomber pour nous les barrières linguistiques avec une bonne grace remarquable. II ressort de ces exposes que le cathétérisme et $\Gamma$ angiocardiographie sont des méthodes indispensables pour une indication opératoire et pour éclairer le chirurgien sur la tactique opératoire. II ressort aussi que Гinformation essentielle du cathétérisme, c'est la mesure de la pression dans Tartère pulmonaire, malgré les difficultés que $\Gamma$ on rencontre pour pénétrer dans ce vaisseau, el les échecs relativement nombreux. Après Гexposé de M. Rossier, nous concevons des doutes sutr la valeur des analyses des gaz du sang et sur la pertinence du calcul des shunts. Le moins que l'on puisse dire, c'est qu'il faut être très prudent dans Tutilisation de ces données. L'angiocardiographie, d'autre part, nous apporte des informations extraordinaires, magnifiquement illustrées par les radio-graphies en série que nous a fait défiler M. Mannheimer. Elle n'est toutefois pas sans danger.

Les deux méthodes ont leurs écueils et leurs avantages. Ce que les orateurs de cette matinee nous ont apporté nous confirme dans le point de vue que j'ai expose dans mon introduction : ce sont des méthodes devenues rapidement

168

Discussion.

indispensables, mais elles doivent être utilisées pour des motifs Men définis, avec discernement et avec le respect de $\Gamma$ être humain.

Discussion de Гexposé de M. McMichacl.

M. Lenègre : L'introduiction de toute nouvelle technique d'exploration dans i'examen clinique des malades doit appeler une critique motivée dont la communication remarquable du Professeur Rossier donne Гexemple.

A ce titre la pratique du cathétérisme des cavités droites du cæur doit faire évoquer certains des accidents que Fon a pu observer à la suite de cette manoeuvre. A ma connaissance trois cas de mort out été signalés : Гun du fait d'une crise angineuse, Гautre à la suite d'une tachycardáe ventriculaire, un troisième par thrombose des veines cathétérisées (fémorale, iliaque, cave) et de Toreillette droite.

Personnellement, sur plus de 700 cathétérismes, nous avons eu à déplorer deux cas de mort, Гum chez une pulmonaire chronique grave qui a succombé en 20 heures du fait d'un collapsus cireulak \&gt; ire mal expliqué ; Гautre chez une femme atteinle d'un rétrécissement mitral cedémaleux des plus sévères, qui a été emportée en quelques heures par un cedème pulmonaire irréductible.

$11 \mathrm{y}$ a lieu aussi de tenir compte de certains chocs par introduction des pyrogènes dans le torrent circulatoire. Ces chocs, que Fon ne peut pas cons-tamment prévenir et qui seraient anodins chez un sujet sain, peuvent devenir redoutables chez certains grands cardiaques : ils élèvent 
brusquement la pres-sion artérielle pulmonaire déjà très haute et peuvent être suivis d'cedème pulmonaire ou de collapsus.

II convient done de s'abstenir momentanément ou définitivement de cathé-tériser les maladies dont la condition cardiaque serait trop précaire.

M. Ferrero : La connaissance des potentiels intra-cardiaques aide à préciser la localisation de Textrémité de la sonde en cours de cathétérisme. Pour préciser si la sonde est immédiatement audessous oui au-dessus des sigmoïdes pulmonaires ou encore à gauche ou à droite d'une cloison intra-auriculaire permeable, les contrôles radioscopiques et manométriques sont parfois insuffisants. La chute énorme des potentiels intra-ventriculaires sitôt les valvul·es pulmonaires franchies ou encore Гonde P intra-auxiculaire « en miroir» de Гoreillette gauche sont d'utiles données pour ces diagnostics.

Le contrôle élect ocardiographique continu au cours du sondage, par les derivations « internes » ou standards, constitue de plus un facteur de sécurité pour le malade.

J. Lequime : A côté du dosage des gaz du sang recueilli par cathétérisme des cavités cardiaques, il existe deux procédés gazométriques susceptibles d'aider grandement au diagnostic des cardiopathies congénítales : la determination du coefficient d'util·isation d'oxygène à $\Gamma$ effort et les mensurations oxymétriques au cours de $\Gamma$ exercice.

On appelle coefficient d'utilisation d'oxygène ou encore « equivalent venti-latoire », le rapport existant entre la consommation d'oxygène el Гair inspire par unite de temps. Chez le sujet normal lors d'une épreuve d'effort de courte durée ce rapport s'accroît ; en effet, la circulation pulmonaire augmente pro-porti onnellement plus que la ventilation. Au cours des cardiopathies congéni-tales s'accompagnant d'un rétrécissement de Fartère pulmonaire (tétralogie de Discussion.

169

Fallot, sténose isolée de Tarlère pulmonaire), le coefficient d'utilisation d'oxy-gène diminue lors de $\Gamma$ exercicc : ce phénomèn $e$ résulle de Гimpossibiliíé dans laquelle se trouve $\Gamma$ artère rétrécie, d'augmenter son diébit d'une façon suffi-sante (Lequime et Denolin). II arrive cependant, dians certains cas de tétra-logie de Fallot, quo les patients réagissent comme des sujets normaux; cela se produit lorsque la circulation coll ·atéral·e est parliculièrement développée ct assure $\mathrm{d} 3 / 8 \mathrm{~s}$ lors une suppléance presque parfaite de Гarlère pulmonaire sténosée. La méthode oxymétrique se prète parfaitement à $\Gamma$ elude des cardiopathies con-génitales : grace à elle, en effet, il est possible d'enregistrer d'une façon continue les variations de la saturation en oxygène du sang artériel « in vivo ; \&gt; . On utilise une cellule pholo-électrique au selenium fixée au lobule de 1'oreille. L·es variations de densité optique survenant à ce niveau lors de changements de composition du sang sont mesurées à $\Gamma$ aide di'un galvanomètre. Chez les sujets atteinls de cardiopathies congénilales à « shunt» vein'o-artériel·, on met en evidence au cours d'une épreuve d'effort une désaturation importanle du sang artériel en oxygène. Ces modifications sont d'autant plus marquees que le « shunt » est plus considerable. De l-elles variations ne s'observent pas chez des sujets normaux ou chez des patients porteurs d'une sténose pulmonaire isolée ou d'une cardiopathie à « shunt » artérioveineux : communication interventri-culaire pure, persistance du canal artériel! (Callebaut, Denolin, Lequime).

Dextrocardie avec situs inversus et malformations cardio-vasculaires associées.

Le catheter pénètre dans une oreillette unique par une veine cave gauche et en sort par une veine cave droite (Denolin et Lequime).

Cardiologia, Vol. XV, 1¹/8sc. 3, 4, 5 (1949)

12 
170 Discussion.

Discussion de Гexposé de M. Mannheimer.

J. Lequime : Dans son rapport, Mi nnheimer a signalé la frequence des malformations cardiovascul aires complexes chez les sujels porteurs d'une dextrocardie. Cetle notion est classique pour ce qui concerne les patients pré-sentant une dextrocardie sans situs inversus visceral. On peut cependant ren-contrer des malformations très complexes au cours de certaines dexlrocardies avec situs inversus complet. Nous avons eu Toccasion d'observer 2 malades cyanosés porteurs d'une dextrocardie avec situs inversus visceral (üenolin et Lequime). Le cathétérisme des cavités cardiaques a montré chez ces sujets Гexis-tence d'une oreillette unique ; en effet, le sang de la cavité auriculaire était pratiquement identique au sang prélevé dans une artère périphérique. De plus, chez Гun d'eux, il exislait 2 veines caves et une sténose de 1.'artère pulmonaire. La radiographie ci-joínte (p. 169) montre le catheter ayant pénétré dans Toreil-lette unique par la veine cave gauche et sorlant de la cavité auriculaire par une veine cave droite. Ces 2 observations sont à rapprocher d'un cas relate par Cournand et d'un cas rapporté par Taussíg.

E. Rossi: Im Zusammenhang mit dem schönen und înteressanten Vortrag Professor Mcnnheimers über die Diagnostik der Pulmoiialstenose möchte ich einige Fälle erwähnen, die wir im Kinderspital Zurich beobachtet haben, und die uns von diesem Standpunkt aus besondere Schwierigkeiten dargebolen haben.

Es handelt sich hauptsächlich um die Fälle von Pulmonalstenose mil Vor-hofseptumdefekt. Bei einem Fall war es uns auf Grund des kliinischen und röntgenologischen Befundes und auf Grund der Druckmessung im re. Herzen nicht möglich, zu entscheiden, $o \cdot b$ eine Falloísche Telrade oder cine Pulmoiialstenose und Vorhofseptumdefekt vorlagen. Sogar die Angiocardiographie, die theoretisch anders sein sollte, hat uns schon im 1.Bilde die Füll ung der Aorta gleichzeitig mit der Pulmonalis gezeigt. Das Kontrastmittel ist vom rechten zum linken Vorhof geflossen, um durch den rechten Ventrikel die Aorta zu erreichen. Es war in diesem Fall nicht möglich, die Sauerstoffsättigung im Blute zu bestimmen. Vielleicht hätte sie uns, wie wir in einem zweiten Falle von Pulmonalstenose und1 Vorhofseptumdefekt feststellen konnten, eine Abgrenzung gegenüber eine Tétrade de Fallot erlaubt. In der ersteren dürfen die Werte im rechten Vorhof, rechten Ventrikel und Pulmonalis nur geringe Schwankungen zeigen. Bei der Tétrade de Fallot dagegen findet man oft höhere Sättigungs-werte in Ventrikel und Pulmonalis als im Vorhof. Was die Bedeulung der Hockerstellung anbetrifft, können wir die Resultate der schwedischen Schule bestätigen, nämlich daß die Hockerstellung kein zu-verlässiges Zeichen ist, um eine mangelhafte von einer zu slarken Durchblutung der Lunge zu unferscheiden. Von unseren 36 Fallen von Tétrade de Fallot zeigten 13 keine Hockerstellung. Dagegen war sie bei 12 Fallen von Eisen-mengerschem Komplex 4 mal zu beobachten.

Discussion de $\Gamma$ exposé de M. Rossier.

Charles Laubry (qui preside) : Avant d'ouvrir la discussion de Гexposé de Rossier, je veux tout d'abord apporter mon salut personnel et celui de la So-ciété Française de Cardiologie à la jeune Société Suisse de Gardiologie. Les remarquables exposes que nous venons déjà d'entendre laissent augurer d'une

Discussion.

171

riche journée scientifique et je forme des væux très chateureux pour nos chers amis suisses qui viennent de nous conférer à mon ami Gallavc rdin el a moi-même un titre qui nous honore grandement. Rossier vient de faire une conference non seulement brillante mais courageuse où il n'a pas crainl de reprendre le problème de l'analyse des gaz du sang jusqu'à la racine et de 
manière extra-ordinairement critique. Les attaques auxquelles il s'est Hvré concernant $\Gamma$ incertitude des résultats obtenus en matière de debit cardiaque m'ont ébranlé et ont sans doule creusé une blessure dans les convictions de beaucoup de ceux qui sont ici presents. A la discussion de panser, si elle le peut, les marques des coups assenés par Rossier. En tout cas c'est d'elle que dépendra le dernier mot.

J.Lequime : Au point de vue technique le dosage des gaz du sang est ex-trémement aisé à Гaide de Гappareil manomélrique de van Slÿke. Cet instrument est à l'heure actuelle d'un emploi p res que universe!. Dans des mains expérimentées il donne des résultats d'une très grande precision. Il- est evident qu'il convient de pratiquer phisieurs determinations sur chaque échantillon de sang. D'autre part ces determinations doivent être effectuées peu de temps après le prélèvement : 2 à $3 \mathrm{~h}$. maximum.

Le rapporteur a souligné Гinlérêt qu'il y avait à determiner la consummation d'oxygène au moment où $\Gamma$ on praliquait le cathétérisme. C'est là une precaution utile. II convient toutefois de faire remarquer que dans Гimmense majorité des cas, la consummation d'oxygène ne paraît pas modifiée d'une façon significative par les manoeuvres de la cathétérisation, en particulier quand il s'agit de grands enfants et d'adultes.

Le rapporteur pense que dans une même cavité cardiaque on peut prélever des échaniilk \&gt; ns de sang ayant des compositions gazométriques très différentes et il émet $\Gamma$ hypothèse que dans une cavité donnée, certaines veines liquid \&lt; es pourraie Пt conserver une veritable indépendance. En réalité, chez un sujet normal des mesures faites aux différents étages d'une même cavité donnent des résultats similaires ou du moins cxtrêmement voisins. S'il existe une communication anormale, il peut arriver, évidemnient, que Гon prélève un échanlillon juste au niveau de $\Gamma$ orifice de communication. Dans de telles conditions il est clair que $\Gamma$ échantill $\cdot$ on recueilli pourra différer du sang prélève dans le reste de la cavité explorée. Lorsque $\Gamma$ on prélève dlu sang dans Гartère pulmonaire, il importe de le faire au niveau du tronc ou à $\Gamma$ origine des branches ; quand le catheter est en effet trop profondément engage dans $\Gamma$ une des branches pulmonaires, il peut arriver que le sang recueilli soit, en fait, du sang artérialisé, ce qui rend les résultats illusoires. D'une façon générale chaque fois qu'un résullat paraissant étrange est observe, il importe de le discuter et de recom-mencer évenluellement le cathétérisme. Les chiffres de debit cardiaque mesures par cathétérisme, sont dans Гensemble très superposables

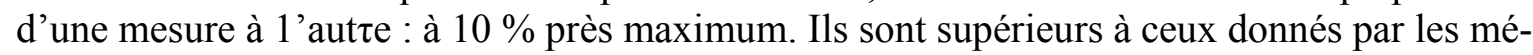
thodes utilisant les gaz étrangers el en particulier par la méthode à Гacétylène.

Pour ce qui concerne la determination dies « shunts », celle-ci peut n'avoir qu'une valeur qualitative et d'indication (canal artériel-, communication inter-auriculaire par exemple). Dans d'autres cas (télralogie de Fallot pure) les résultats observes sont d'une très grande precision et superposables d'une mesure à $\Gamma$ autre. Cette recherche quantitative du « shunt.» dans la maliadie de Fallot peut avoir une très grande valeur pratique. Si on observe, en effet, chez un

$12^{*}$

172

Discussion.

sujel donné, une circulation pulmonaire diflérant assez peu de la circulation générale et urn « shunt » veino-arlériel faibl·e, une intervention chirurgicale n'est pas indiquée. Au contrairc si la circulation pulmonaire est considérable-ment réduite, une operation du type Blalock-Taussig ou du type Potts rendra de grands services ; encore convicnt-il dans de tels cas de s'efforcer d'avoir une idee de la valeur de la circulation collatérale suppléant Tartère pulmonaire sténosée. Cette 
recherche est possible par des procédés gazométriques. Au cas où la circulation collatérale est très importante, Fopération doit-être dé-conseillée.

La determination des gaz du sang doit être complétée par des mensurations des pressions inlracardiaques. 11 existe à Theure actuelle d'excellents mano-mètres électriques (Hamilton, Warburg, Sandborn) permettant des mensurations très exactcs. II imporle aussi quo Гexamen soit complete par Гangio-cardiographie. L.' association de ces divers procédés d'examen permetlra, d'ans $\Gamma$ immense majorité des cas, d'arriver à un diagnostic exact.

McMichael: I agree with Professor Rossier that it is not possible to give an exact figure for basal cardiac output. What one measures is cardiac output at the time of the examination. With complicated procedures like cardiac catheterisation it is impossible in conscious patients to have strictly basal conditions. It is better to express cardiac output simply as the figure obtained at that particular time.

From observation of the oxygen unsaturation of blood samples obtained in pairs from the right ventricle and also from the right ventricle and the right auricle, we find that the coefficient of variation of the arleriovenous oxygen difference is \pm 3 per cent. The oxygen consumption done before and after the catheterisation procedure has shown a coefficient of variation of \pm 5 per cent. When these are combined we believe that the standard error of a cardiac output estimation by means of catheterisation is about \pm 6 per cent. Estimations differing by 12 per cent will therefore be significant. Very occasionally samples may be drawn from the coronary sinus which are grossly reduced in oxygen content. These are easily recognised and should, of course, not be used for cardiac output determination.

In our laboratory we use a water immersed Haldane blood gas apparatus for the direct estimation of the amount of oxygen taken up by a measured sample of blood from the right heart on shaking in air. This is practically a direct measurement of the amount of oxygen taken up by such a sample on passage through the lungs. We do not use the Haldane apparatus for the estimation of the oxygen capacity. We are fully aware of the difficulties of obtaining this figure which have been emphasised by Professor Rossier. This estimation, however, is not necessary for cardiac output determination. It is interesting that the catheterisation technique checks so closely with estimations of cardiac output made by other methods such as Hamilton's dye method. We would also agree with Professor Rossier that where arteriovenous differences become very small, the calculation of cardiac output is open to very serious error. The calculation of the volume of blood shunted from one side of the heart to another in congenital heart disease is also a procedure fraught with considerable possibility of error due to incomplete mixing of two blood streams grossly differing in composition.

Discussion.

\section{3}

Aloys Midler: Aus den heutigen Vorträgen geht hervor, daß der Herz-kathelerismus, sachverständig ausgeführt, ein relativ harmloser Eingriff ist, der zur Diagnose bes·timmter Krankheilen nicht nur ausgeführt werden darf, son-dern nofwendig ist. Es ist erfreul-ich, daß der mutige Selbstversuch des d amals jnngen Forschers Forßmann lieute naeh ca. 20 Jahren seine Früchte trägt. Die mit dieser Methode gewonnenen Erkenntnisse sind auch für diie all $\cdot$ gemeine Kreislauflehre wertvol·1. Aus den Füllungsbildern, die mis Herr Kollege Mann-heimer gezeigt hat, läßt sich bereils ersehen, daß die Strömungsverhältnisse im kleinen Kreislauf unseren heutigen Anschauungen entsprechen. Die Methode kann bei gewisser Änderung der Technik noch leistungsfähiger werden. 
Was nun die Druckmessungen in den Herzventrikeln anbelangl, wird deren Bedeutung überschälzt. Die von Herrn Kollegen Rossi gezeigten Kurven sind sicher entstellt. Es riihrt dies z. T. daher, daß die wirksame Masse durch Ansctzen des langen Katheters sehr groß wird. Häui'ig ist auch die Füllung des Systems nicht vollsíändig. Man darf nicht vergessen, daß geringsle Mengen vor-hamdtener Luft den Volumelastizitätsmodul sehr stark ändern. Beide Faktoren wirken sich auf die Frequenz ungünstig aus. Ich bin überzeugt, daß die meisten hiefür veiwendeten Manometer nur eine Frequenz von 10 bis 15 Sek. aufwiesen. Obschon eine einwandfrcie Druckmessung im Herzen auf diese Art unmöglich ist, möchte ich den erhaltenen Kurven nicht jeden Wert für die Klinik ab-sprechen. Auf jeden Fall muß aber vcrlangt werden, daß die Frequenz vor und nach dem Versuch experimentel! ermiltelt wird. Es läßt sich diann eine evtl. Korreklur vornehmen.

Die große Skepsis des Herrn Kollegen Rossier gegenüber malhematischen Formeln ist unberechtigt und iingerecht. Es ist leider eine uinbegreifliche Tatsache, daß schon der Medizinstudent eine starke Abneigung gegen mathe-malische Formulierungen zeigt, komplizierte chemische Formeln aber wider-standslos auswen - dig 1·ernt. In Wirklichkeit ist die mathematische Formel die einfachste, genauesle und klarsle Ausdrucksweise eines Gesetzes. Wenn ex-perimentelle Ergebnisse mit gegebenen Formeln nicht übereinstimmen, liegt die Schuld nicht an der Formel, sondcrn an demjenig·en, der sie anwendet. Mil großer Vorl-íel \&gt; e entlehnen Biologen gewisse Formeln aus der Physik und Technik, um sie für Vorgänge anzuwenden, für die sie nicht aufgestellt sind.

Der Vorstellung, die sich Herr Koll·ege Rossier über die Strömungsverhältnisse macht, kann ich nicht folgen. Eine Art Stromfädenbündehmg, die sich durch den Zufluß auf dem Venenwege bildet und trotz der Passage durch das Пerz bis in die Pulmonalis erhält, ist nicht denkbar. Die Versuche von W. R. Heß, die er zum Beweise zitiert, dürfen nicht auf diese Verhältnisse überlragen werden. Hingegen ist eine Stagnation gewisser Randzonen des Blutes infol'ge der unregelmäßigen Wandbegrenzung der Herzhöhlen möglich. Dadurch könncn Differenzen im Sauersíoffgehall auftreten.

E. Mannheimer: The splendid paper given by Professor Rossier is of the greatest importance since it shows how careful we all must be in judging the results of blood gas analysis in heart catherizalion. Lagerlöf, Werkö and myself have stressed the same point and all who work with cathelerizations have to take into consideration the limitations of the method and the sources of errors. But we know also that in many cases the figures tha,t we obtain can be of clinical use. There is an expression: «Radiograms never lie, the radio174

Discussion.

logists sometimes do.» This sentence can also be applied to heart catheterization.

P. H. Rossier: Je partagc entièrement 1'opinion du Prof. A. Mailer con-cernant $\Gamma$ 'impo $\Gamma$ tance des formules mathématiques en biologie et en médecine. Comme je Гai fait remarquer au cours de mon expose, tout depend ce pendant de la manière dont on utilise les formules qui sont à notre disposition. On ne peut mettre en doute que \&lt; les formules proposées en vue de la determination quantitative de Timportance des shunts sont exactes, mathématiquement par-lant. Malheureusement, le manque d'homogénéité du sang intra-cardiaque in-troduit une cause d'erreur importante, si importante parfois que tous les cal-culs deviennent illuisoires. Quant à ce manque dl'homogénéité, il peut s'expliquer si $\Gamma$ on $\mathrm{ad}^{3} / 8$ net $\Gamma$ exislence. de courants laminaires dans le cæur, courants dont la réalité a été démontrée par Hess au niveau des artères. Si les physiologistes peuvent expliquer le manque d'homogénéité du sang intra-cardiaque par une autre 
théorie, je suis prêt à Гaccepter à condition qu'elle rende compte des faits expérimentaux d'une manière plus satisfaisante que Thypothèse que je propose.

Je remercie le Prof. McMichael et le D" Lequime de leurs remarques, mais ma reconnaissance va avant tout au Dr Mannheimer qui vient de vous montrer que ses determinations de debit cardiaque sont entachées d'erreurs expéri-mentales encore plus considerables que les miennes. Ne nous a-t-il pas dit, en effet, que ses determinations de conlenu d'oxygène du sang intracardiaque comportent une erreur systématique de $+1,2$ vol. $\%$ - ce qui entraîne une erreur dans l'estimation du debit cardiaque de $\Gamma$ ordre de $\pm 1.000 \mathrm{cc}$, si je cal-cule bien...

Discussion de $\Gamma$ exposé de M. Rossi.

E. Mannheimer .- Je vous félicite, D1' Rossi, de vos magnifiques résultats. Nous avons trouvé très souvent des « squatters » parmi les cas d'Eisenmenger. Le pourcentage dies « squatters » de nos cas de $\Gamma$ hôpilal Lovisa concernant la maladie bleue a été :

parmi les cas de la télrade de Fall·ot : $72 \%$; parmi les cas du complexe d'Eisenmenger : $30 \%$. V. Puddu : L'injection intraveineuse d'élher peut être utile chez des enfants pour démontrer un shunt droit-gauche grace à la reaction du picotement cutané qu'elle provoque dans ces cas. Grob: Dr. Rossi hat auf die verschiedienen Herzkonfigurationen bei der Tetralogie von Fallot aufmerksam gemacht. Diese verschiedenen Formen haben nicht nur akademisches Interesse, sondern sind z. T. besonders bezüglich der operativen Indikalion von praktischer Bedeulung. Eine besondere Gruppe bilden die Fälle mit extremer Dextro posit ion der Aorta, die eine Zwischenstufe zwischen den gewöhnlichen Fallot und dier Pseudoform des Truncus arteriosus darstellen. Ich darf vielleicht an Hand einiger Diapositive nochmals die Cha-rakteristika dieser Form herausheben. Diese Fälle zeichnen sich aus: 1. durch einen auffallend großen Aortenbogen, der in der a.-p.-Aufnahme den Rand der Wirbelsäule rechterseits und linkerceils überragt; 2. durch eine auffallend starke Hebung der Herzspitze als Ausdruck der starken Rechtshypertrophie. Es sind dies die ausgesprochensten \&lt; .sabots»; 3. durch eine sehr starke Kon-kavilät des Pulmonalisbogens, die durch den überragenden Aortenknopf noch akzentuiert erscheinl; im Angiocardiogramm erscheint der Aortenbogen auffallend breit. Infolge der extremen Dextroposition hat er sich in die Frontal-

Discussion. 175

ebene eingestellt. 5. Das Kaliber der Aorta ist besonders im Bereiche der Aorta ascendens und des Aortenbogens auffallend mächtig.

Anatomisch sind diese Fälle der Tetral \&lt; ogie von Fallot zuzuschreiben, denn sie weisen ihre 4 Kardinalsymptome auf, ftmktionell aber slehen sie dem Pseudotruncus näher und sind 1 in therapeutischer Hinsicht wie dieser operativ ein noli me tangere, worauf Dr. Rossi hingewiesen hat. Ergänzend möchte ich noch beifügen, daß diese Fälle eine ausgesprochene Hypoplasie der Arteria puhnonclís zeigen, wie wir dies in verschiedenen Fallen feststellen konnten und auch aus diesem Grunde für eine Gefäßanastomose nach Blalock oder Potts ungünstige Verhällnisse aufweisen. Daß diese Fälle dem Pseudotnincus arteriosus naheslehen, geht auch daraus hervor, daß oft vermehrte und er-weilerte lironchialarte $\tau$ ien vorhanden sind. (Demonstration eines Falles mit großer retrooesophagealer Eindellung durclı eine erweiterte Bronchialarterie.) Diese Fälle zeigen, wie fließend die Übergänge zwischen den anatomischen Formen bei den kongonitalen Vitien sein können.

VI

(Université de Bruxell·es. Clinique médicale de FHôpilal St-Pierre ct Groupement scientifique pour 1 'etude et $1 \cdot e$ traitement des Cardiopathies congénitales et acquises.) 
Le phénomène du « Squatting » au cours des cardiopathies congénitales.

Par J. LEQUIME, C. CALLEBAUT et H. DENOL·IN.

Le phénomène de Гaccroupissement (squatting) est tm phénomène bien connu de tous ceux qui s'intéressent aux cardiopathies congénitales. On sait que très souvent les patients atteints de malformations cardiaques avec cyanose prennent avec predilection la position accroupie : celleci parait les placer dans une situation plus confortable que n'importe quelle autre ; certains sujets Tadoptent même en dormant.

Une premiere remarque est à faíre : tous les malades porteurs d'une malformation cardiaque cyanogène ne font pas du « squatting ». Mannheímer (1949) note ce phénomène chez $81,6 \%$ des sujets présentant une tétralogie de Fallot et chez $43 \%$ des patients avec complexe d'Eisenmenger. Donzelot (1949) Гobserve chez $85 \%$ des cyanosés congénitaux ; nous Гavons relevé dans $80 \%$ de nos cas. II n'est nullemént exceptionnel de voir des congénitaux parfois très cyanosés ne pas s'accroupir. Une seconde remarque est importante : le " squatting " possède de nombreux equivalents, véritables attitudes de repos : certains patients pre- 